\title{
Identification of Common Oncogenic Genes and Pathways Both in Osteosarcoma and Ewing's Sarcoma Using Bioinformatics Analysis
}

Jingwei Zhang

Shanghai Fengxian Central Hospital https://orcid.org/0000-0002-1083-5023

Wenjun Liu

Shanghai Fengxian Central Hospital

Liang Ding

Shanghai Fengxian Central Hospital

Dongdong Cheng

Shanghai Sixth Peoples Hospital

Haijun Xiao ( $\nabla$ orthopaedicsfx@163.com )

Shanghai Fengxian Central Hospital

\section{Primary research}

Keywords: osteosarcoma, Ewing's sarcoma, differentially expressed genes, protein-protein interaction, hub genes

Posted Date: April 13th, 2021

DOI: https://doi.org/10.21203/rs.3.rs-358166/v1

License: (c) (1) This work is licensed under a Creative Commons Attribution 4.0 International License. Read Full License 


\section{Abstract}

Objective: This study aimed to explore common oncogenic genes and pathways both in osteosarcoma and Ewing's sarcoma.

Methods: Microarray data were obtained from the Gene Expression Omnibus (GEO) database. Differentially expressed genes (DEGs) were respectively identified using the limma package, followed by intersection of common DEGs. Then, protein-protein interaction (PPI) networks were constructed and hub genes were identified. Furthermore, functional enrichment analysis was analyzed. The expression of common oncogenic genes was validated in 38 osteosarcoma and 17 Ewing's sarcoma tissues by RTqPCR and western blot.

Results: 201 genes were differentially expressed. There were 121 nodes and 232 edges in the PPI network. 12 genes were considered as hub genes. Functional enrichment analysis results showed that hub genes FN1, COL1A1 and COL1A2 were all involved in extracellular matrix, protease binding and ECMreceptor interaction, which could be involved in the development of osteosarcoma and Ewing's sarcoma. Among common oncogenic genes, FN1, COL1A1 and COL1A2 were lowly expressed both in osteosarcoma and Ewing' s sarcoma tissues at mRNA and protein levels.

Conclusion: Our findings revealed that common oncogenic genes such as FN1, COL1A1 and COL1A2 and pathways were both in osteosarcoma and Ewing's sarcoma.

\section{Introduction}

Osteosarcoma and Ewing's sarcoma are the two most common primary bone malignancies in children and adolescents [1, 2]. Although therapeutic strategies have made great progress, patients' survival time has only improved marginally $[3,4]$. The high morbidity and mortality of these two diseases require more research to characterize and understand their potential molecular mechanisms $[5,6]$. The molecular pathways involved in these two diseases will facilitate better treatments. Increasing evidence suggests that many cellular signaling molecules are involved in tumorigenesis, and many specific targets have been identified based on these signaling pathways [7, 8]. Furthermore, at the molecular levels, a common feature of these two diseases is a change in gene expression. Knowledge of the molecular pathogenesis of osteosarcoma and Ewing's sarcoma is still limited, and good diagnostic and prognostic tools are still lacking.

To analyze the pathogenesis of osteosarcoma and Ewing's sarcoma, multiple DEGs have been identified between cancer tissues and normal samples among the population using multiple methods $[9,10]$. Because of the large individual differences and heterogeneity of these two diseases, DEGs identified may not accurately describe a single disease sample [11-13]. Moreover, it is difficult to compare gene expression measurements from different samples and platforms [14]. It has been suggested direct comparisons of gene expression levels between diseased tissues and adjacent normal tissues [14]. However, it lacks proper statistical control, and its applications are limited because corresponding normal 
tissue specimens are usually not available. Therefore, unlike previous studies, this study aimed to identify key genes and pathways common in osteosarcoma and Ewing's sarcoma using integrated bioinformatics methods.

\section{Results}

\section{Identification of DEGs and their biological functions for osteosarcoma}

Using the limma package, DEGs with $|\log F C|>1.5$ and $P<0.05$ were screened between 5 cases of osteosarcoma cells and 1 case of mesenchymal stem cell from the GSE70414 dataset. As a result, a total of 329 DEGs were identified for osteosarcoma, including 75 up-regulated and 254 down-regulated genes. The expression patterns of these genes between mesenchymal stem and osteosarcoma cells were shown in Fig. 1A. Supplementary Table 1 listed the top ten up-regulated genes for osteosarcoma cells, such as LOC728613, ZIC2 and CD24. Furthermore, the top ten down-regulated genes for osteosarcoma were listed in Supplementary Table 2.

To explore potential biological functions and pathways of the 329 DEGs in osteosarcoma cells, functional enrichment analysis was performed using the cluster Profiler package. GO terms included biological process (Fig. 1B), cell component (Fig. 1C) and molecular function (Fig. 1D). KEGG results showed that these genes were mainly enriched in several pathways related with cancer, such as microRNAs in cancer and p53 signaling pathway (Fig. 1E). The detailed information about KEGG pathway was listed in Supplementary Table 3. Based on these osteosarcoma-related DEGs, GSEA was also presented. Our data suggested that cancer metastasis-related biological processes were significantly enriched, such as cell motility (Fig. 1F), extracellular matrix (Fig. 1G), signaling receptor binding (Fig. 1H) and regulation of cell adhesion (Fig. 11).

We further probed into the biological functions of up- and down-regulated genes, respectively. The data showed that up-regulated genes were distinctly involved in regulation of binding (Fig. 2A), anchored component of external side of plasma membrane (Fig. 2B), ubiquitin (Fig. $2 \mathrm{C}$ ) and TGF- $\beta$ signaling pathway (Fig. 2D). Down-regulated genes could significantly participate in regulating extracellular structure (Fig. 2E), extracellular matrix (Fig. 2F), cell adhesion molecule binding (Fig. 2G) and focal adhesion (Fig. 2H).

\section{Identification of DEGs and their biological functions for Ewing's sarcoma}

We further analyzed DEGs between 5 cases of Ewing's sarcoma cells and 1 case of mesenchymal stem cell from the GSE70826 dataset. A total of $1688 \mathrm{DEGs}$ with $|\operatorname{logFC}|>1.5$ and $\mathrm{P}<0.05$ were identified between Ewing's sarcoma cells and mesenchymal stem cells, composed of 700 up-regulated and 988 down-regulated genes (Fig. 3A). The top ten up-regulated and down-regulated genes were listed in 
Supplementary Table 4 and Supplementary Table 5, respectively. The potential functions and pathways of DEGs for Ewing's sarcoma cells were explored using the cluster Profiler package. The GO results showed that these genes were significantly related to extracellular matrix (Fig. 3B), cell adhesion molecular binding (Fig. 3C), and extracellular structure organization (Fig. 3D). Furthermore, these DEGs were mainly involved in several KEGG pathways associated with cancer, like PI3K-Akt signaling pathway, TGF- $\beta$ signaling pathway and so on (Fig. 3E). The genes in the KEGG pathways were listed in Supplementary Table 6. GSEA results also demonstrated that extracellular matrix (Fig. 3F), collagen containing extracellular matrix (Fig. 3G), DNA binding transcription factor activity (Fig. $3 \mathrm{H}$ ) and cell substrate adhesion (Fig. 3I).

The biological functions of up- and down-regulated genes were separately analyzed for Ewing's sarcoma. Our data suggested that up-regulated genes were mainly enriched in axon development (Fig. 4A), neuronal cell body (Fig. 4B), transcription (Fig. 4C) and signaling pathways regulating pluripotency stem cells (Fig. 4D). Meanwhile, down-regulated genes exhibited a significant correlation with extracellular structure organization (Fig. 4E), extracellular matrix (Fig. 4F), cell adhesion molecular binding (Fig. 4G) and PI3K-Akt signaling pathway (Fig. 4H).

\section{Common DEGs both in osteosarcoma and Ewing's sarcoma compared to mesenchymal stem cells}

We comprehensively analyzed the common DEGs between osteosarcoma and Ewing's sarcoma. A total of 201 genes were differentially expressed both in osteosarcoma and Ewing's sarcoma compared to mesenchymal stem cells (Supplementary Fig. 1A). Heatmap depicted the difference in expression pattern of these common DEGs between osteosarcoma cells (Supplementary Fig. 1B) and Ewing's sarcoma cells (Supplementary Fig. 1C) compared to mesenchymal stem cell, respectively.

\section{PPI networks of common DEGs both in osteosarcoma and Ewing's sarcoma}

To explore relationships between these common DEGs, the 201 DEGs were analyzed by the STRING. Then, a PPI network was constructed and visualized using the Cytoscape. There were 121 nodes and 232 edges in the PPI network (Supplementary Fig. 2A). The genes with degree $>10$ were considered as hub genes (Supplementary Table 7). In addition, a subnetwork was then constructed using the Cytoscape MCODE, composed of 12 nodes and 51 edges (Supplementary Fig. 2B). The hub genes in the network were all down-regulated both in osteosarcoma and Ewing's sarcoma cells compared to mesenchymal stem cells.

\section{Functional enrichment analysis of common DEGs both in osteosarcoma and Ewing's sarcoma}

The cytoscape plugin ClueGO was used to visualize the functional enrichment analysis results of common DEGs both in osteosarcoma and Ewing's sarcoma compared to mesenchymal stem cells. 
Biological processes enriched by these genes were shown in Supplementary Fig. 3, such as sprouting angiogenesis and regulation of cell-substrate adhesion. Cell component results showed that these genes were mainly enriched in collagen-containing extracellular matrix, extracellular matrix, extracellular matrix component, platelet alpha granule lumen and so on (Supplementary Fig. 4). As for molecular function, these genes were significantly associated with protease binding, collagen binding, heparin binding, integrin binding and metalloendopeptidase activity (Supplementary Fig. 5). KEGG pathway enrichment analysis results showed that these genes were mainly involved in ECM-receptor interaction (including FN1, COL1A1, COL1A2, COMP, ITGA5 and THBS1) and AGE-RAGE signaling pathway in diabetic complications (including FN1, COL1A1, COL1A2, SERPINE1 and AGTR1) in Supplementary Fig. 6.

\section{Validation of common oncogenic genes in osteosarcoma and Ewing's sarcoma tissues}

Common oncogenic genes were further validated in 38 osteosarcoma and 17 Ewing's sarcoma tissues by RT-qPCR and western blot. Our RT-qPCR results showed that FN1 (Fig. 5A), COL1A2 (Fig. 5B), COL1A1 (Fig. 5C) and ADAMTS2 (Fig. 5D) were down-regulated both in osteosarcoma and Ewing's sarcoma tissues compared to normal tissues. There was no significant difference in TIMP1 expression between osteosarcoma and normal tissues. But TIMP1 was lowly expressed in Ewing's sarcoma tissues than normal tissues (Fig. 5E). In Fig. 5F, THBS1 had a lower expression in osteosarcoma than normal tissues. No significant difference was detected between Ewing's sarcoma tissues than normal tissues. Furthermore, POSTN exhibited a significantly higher expression in osteosarcoma not Ewing's sarcoma tissues than normal tissues (Fig. 5G). ITGA5 was markedly down-regulated in osteosarcoma tissues but up-regulated in Ewing's sarcoma tissues compared to normal tissues (Fig. 5H). However, no significant difference was found in SERPINE1 and TIMP3 expression between osteosarcoma or Ewing's sarcoma tissues and normal tissues (Fig. $5 \mathrm{I}, \mathrm{J}$ ). Our western blot results confirmed that FN1, COL1A2 and COL1A1 proteins were significantly lowly expressed in osteosarcoma or Ewing's sarcoma tissues than normal tissues (Fig. 5K-N).

\section{Discussion}

The cell line panel provides a valuable model system for analyzing gene expression in Osteosarcoma and Ewing's sarcoma. In this study, a comprehensive bioinformatics approach was used to analyze gene expression in Osteosarcoma and Ewing's sarcoma cell lines compared to normal controls. We identified 329 DEGs with $|\log F C|>1.5$ and $P<0.05$ in 5 cases of osteosarcoma cells compared to 1 case of mesenchymal stem cell using the limma package, including 75 up-regulated and 254 down-regulated genes. Among 75 up-regulated genes, the top ten genes according to fold change included LOC728613, ZIC2, CD24, ABLIM1, MYLIP, SIPA1L2, RHPN2, S100A4, LHX2 and LOC100996740. Furthermore, the top ten down-regulated genes included FBLN5, PLD5, TRIM22, FN1, KCTD12, CTSK, ABI3BP, HAS2, POSTN and TACSTD2. Functional enrichment analysis results revealed that these DEGs were involved in several pathways related with cancer, such as microRNAs in cancer and p53 signaling pathway. We found that BCL2L11, E2F2, FOXP1, HMOX1, ITGA5, MIR34A, MARCKS, ZEB1, THBS1, TIMP3, VIM, RPS6KA5 were 
enriched in the microRNAs in cancer pathway. It has been recognized that miRNAs, a class of small noncoding RNA, are involved in tumorigenesis and development of various cancers including osteosarcoma by regulating protein expression at the post-transcriptional level $[17,18]$. Furthermore, DDB2, IGFBP3, SERPINE1, THBS1 and TP53I3 were enriched in p53 signaling pathway. Increasing evidence suggests that abnormal expression of many genes could activate p53 signaling pathway in osteosarcoma $[19,20]$. Thus, the above genes might be involved in the development of osteosarcoma, which require further experimental validation.

Similarly, we analyzed DEGs in 5 cases of Ewing's sarcoma cells compared to 1 case of mesenchymal stem cell. A total of 1688 DEGs were identified, including 700 up- and 988 down-regulated genes. The top ten up-regulated genes were as follows: COL6A3, COL8A1, CTHRC1, SRGN, TGFBI, MICAL2, ITGBL1, HAS2, LGALS3 and SERPINE1. Down-regulated genes included COL6A3, COL8A1, CTHRC1, SRGN, TGFBI, MICAL2, ITGBL1, HAS2, LGALS3 and SERPINE1. Functional enrichment analysis results showed that these DEGs were mainly involved in several KEGG pathways associated with cancer, like PI3K-Akt signaling pathway and TGF- $\beta$ signaling pathway. Activation of PI3K-Akt signaling pathway may contribute to the development of Ewing's sarcoma [21, 22]. TGF- $\beta$ signaling pathway could inhibit apoptosis, and promote proliferation for Ewing's sarcoma cells [23]. Genes involved in the TGF- $\beta$ signaling pathway might promote the progression of Ewing's sarcoma.

To identify common oncogenic genes both in osteosarcoma and Ewing's sarcoma, common DEGs both in osteosarcoma and Ewing's sarcoma were intersected. A total of 201 genes were identified. Heatmap depicted most of these genes had similar expression pattern both in osteosarcoma and Ewing's sarcoma cells compared to mesenchymal stem cells, indicating that these genes might be common oncogenic genes for osteosarcoma and Ewing's sarcoma. However, further analysis needs to be performed. Afterwards, A PPI network based on these common genes was constructed. A hub gene plays a vital role in biological processes. In related pathways, the regulation of other genes is often dominated by this gene. The 12 genes with degree $>10$ were considered as hub genes. Intriguingly, these hub genes were all down-regulated both in osteosarcoma and Ewing's sarcoma cells compared to mesenchymal stem cells. The 12 hub genes were as follows: FN1, COL1A2, COL1A1, POSTN, TIMP1, THBS1, SERPINE1, ITGA5, TIMP3, ADAMTS2, MMP13 and COMP. We further confirmed the expression patterns of these hub genes between osteosarcoma or Ewing's sarcoma tissues and normal tissues by RT-qPCR. Functional enrichment analysis results of common DEGs showed that these genes were mainly involved in ECMreceptor interaction (including FN1, COL1A1, COL1A2, COMP, ITGA5 and THBS1) and AGE-RAGE signaling pathway in diabetic complications (including FN1, COL1A1, COL1A2, SERPINE1 and AGTR1). We found that hub genes FN1, COL1A2 and COL1A1 were involved in the two pathways. Our western blot confirmed that FN1, COL1A2 and COL1A1 were lowly expressed both in osteosarcoma and Ewing's sarcoma tissues compared to normal tissues. The composition and structure of ECM is known to be a key determinant of tumor metastasis. Recent studies have reported that the activation of Wnt / $\beta$-catenin promotes the secretion of ECM proteins in tumor cells [24]. Consistent with previous studies, in this study, ECM-receptor interaction was highly enriched by DEGs in osteosarcoma cells [25]. FN1 has been confirmed to be involved in ECM-receptor interaction [26]. It is up-regulated in the chemo-resistant osteosarcoma cell lines 
and tissues and associated with poor prognosis $[27,28]$. Our results showed that COL1A1 was downregulated in osteosarcoma cells, which was consistent with previous study [29]. Furthermore, it has been reported that in the osteosarcoma cells, COL1A1 and FN1 could be associated with gastric cancer prognosis [30]. A previous study has found that COL1A2 and COL1A1 could be associated with TWIST1, a key transcription factor in metastasis [31]. Our GO enrichment analysis results showed that FN1, COL1A2 and COL1A1 were significantly enriched in extracellular matrix, protease binding and so on, indicating that the three genes could play a critical role in the development of osteosarcoma and Ewing's sarcoma.

In summary, the observation of common key genes in Osteosarcoma and Ewing's sarcoma suggests that these specific genetic changes may be involved in regulation of the progression of Osteosarcoma and Ewing's sarcoma. These hub genes can be used as candidate targets for the diagnosis and treatment of Osteosarcoma and Ewing's sarcoma.

\section{Conclusions}

To explore possible common oncogenic factors both in osteosarcoma and Ewing's sarcoma, we comprehensively analyzed the mRNA expression pattern in osteosarcoma cells and Ewing's sarcoma cells. Common key genes both in Osteosarcoma and Ewing's sarcoma were identified, such as FN1, COL1A2 and COL1A1, which required further validation.

\section{Materials And Methods Microarray data}

GSE70414 and GSE70826 microarray datasets were downloaded from the GEO (http://www.ncbi.nlm.nih.gov/geo/) database. GSE70414 dataset contains the mRNA expression data of five osteosarcoma cells and one human mesenchymal stem cell. GSE70826 microarray dataset contains the mRNA expression data of five Ewing's sarcoma cells and one human mesenchymal stem cell. The two datasets are based on the GPL570 platform. The expression levels of probes mapping into multiple genes were averaged.

\section{Analysis of DEGs}

According to the expression profiling data of GSE70414 and GSE70826, DEGs in osteosarcoma or Ewing's sarcoma cell lines compared with human mesenchymal stem cells were identified using the Limma package (http://www.bioconductor.org/packages/release/bioc/html/limma.html). Genes with $\mid \log$ fold change $(\mathrm{FC}) \mid>1.5$ and $\mathrm{P}<0.05$ were considered as DEGs. Unsupervised hierarchical clustering of different samples was performed using the R package based on microarray data.

\section{Functional enrichment analysis}


Functional enrichment analysis of DEGs was performed using the $\mathrm{R}$ language clusterProfiler package. Gene Ontology (GO) terms were significantly enriched by DEGs. GO analysis includes biological process, cellular component, and molecular function. The number of DEGs involved in GO terms was counted. Furthermore, we made Kyoto Encyclopedia of Genes, and Genomes (KEGG) analysis, which was used to find the pathway terms involved in DEGs. P-value $<0.05$ was considered significantly enriched. The results were visualized using the cytoscape plugin ClueGO. Gene Set Enrichment Analysis (GSEA) was also performed, with the threshold of 1,000 permutations and a false discovery rate (FDR) $<0.25$.

\section{PPI network analysis}

A PPI network was constructed by the STRING online database (http://string-db.org/) to predict the relationships among the products of differentially expressed genes [30]. The relationships of DEGs were visualized by use of Cytoscape (version 3.4.0) [31]. Nodes stand for biological molecules and edges connected the nodes represent their interactions [14]. Furthermore, using the Cytoscape plugin MCODE, the most significant module was screened in the PPI network.

\section{Patients and specimens}

38 osteosarcoma and 17 Ewing's sarcoma patients who underwent complete resection were recruited from Shanghai Sixth People's Hospital between January 2014 and December 2015. The tumor tissue and the matched adjacent normal tissue were simultaneously collected from each patient. None of them experienced chemotherapy before surgery. This study gained the approval of the Ethical Committee of Shanghai Sixth People's Hospital, strictly following the Declaration of Helsinki(YS-2018-039). Each participant provided written informed consents. All the resected specimens were placed instantly into liquid nitrogen and stored at $-80^{\circ} \mathrm{C}$. Table 1 listed the clinical characteristics of patients with osteosarcoma and Ewing's sarcoma. No significant differences in age, gender, and death were found between the two groups. But there was a significant difference in recurrence between the two groups $(p=$ 0.0406). 
Table 1

The clinical characteristics of patients with osteosarcoma and Ewing' s sarcoma.

\begin{tabular}{|llll|}
\hline Parameters & Osteosarcoma $(\mathbf{N}=\mathbf{3 8})$ & Ewing' s sarcoma $(\mathbf{N}=\mathbf{1 7})$ & P value \\
\hline Age(year) & & & 0.3711 \\
\hline$\leq 18$ & 27 & 10 & \\
\hline$>18$ & 11 & 7 & 0.9378 \\
\hline Gender & & & \\
\hline Male & 25 & 11 & \\
\hline Female & 13 & 6 & 0.0406 \\
\hline Recurrence & & & \\
\hline Yes & 6 & 7 & 0.0550 \\
\hline No & 32 & 10 & \\
\hline Death & & & \\
\hline Yes & 10 & 9 & \\
\hline No & 28 & & \\
\hline
\end{tabular}

Total RNA was extracted from tissues utilizing TRIzol (Invitrogen, Carlsbad, California, USA), which was reverse transcribed into CDNA via the reverse transcriptase kit (Invitrogen). PCR was presented with the TB Green ${ }^{\circledR}$ Premix Ex Taq ${ }^{\text {TM }}$ II kit (TAKARA, Japan) according to the following procedures: 40 cycles of $94^{\circ} \mathrm{C}$ lasting $15 \mathrm{~s}, 60^{\circ} \mathrm{C}$ lasting $10 \mathrm{~s}$, and $72^{\circ} \mathrm{C}$ lasting $20 \mathrm{~s}$. Gene expression was normalized to GAPDH, followed by calculation of relative expression levels with the $2^{-\Delta \Delta C t}$ method. The primer sequences were listed in Supplementary Table 8.

\section{Western blot}

Tissues were lysed by RIPA lysate (Beyotime, Beijing, China) on the ice, which were centrifugated at $12000 \mathrm{~g}$ for $12 \mathrm{~min}$. Afterwards, the supernatant was harvested. BCA kit (Beyotime) was utilized to determine the protein concentration. Proteins were separated via SDS-PAGE, followed by being transferred onto PVDF membranes. After being blocked by $0.5 \%$ skimmed milk lasting $2 \mathrm{~h}$ at room temperature, the membranes were incubated with primary antibodies against FN1 (1/1000; ab199056), COL1A1 (1/1000; ab255809), COL1A2 (1/1000; ab96723) GAPDH (ab18160) at $4{ }^{\circ} \mathrm{C}$ overnight, followed by secondary antibodies (1/5000; ab7090) at room temperature for $2 \mathrm{~h}$. Immunoreactivities were assessed via the Western Lighting Ultra (Thermo Fisher Scientific, USA).

\section{Statistical analysis}

Statistical analysis was carried out via R 3.6.3 and GraphPad 7.0. Data from experiments are expressed as means \pm standard deviation. Differences in clinical features between osteosarcoma and Ewing's 
sarcoma groups were assessed by Chi-square test. Paired student's t test was used for comparisons between two groups, while multiple comparisons were presented via one-way analysis of variance. Differences with $p<0.05$ was considered statistically significant.

\section{Abbreviations}

GEO: Gene Expression Omnibus; FC: fold change; GO: Gene Ontology; KEGG: Kyoto Encyclopedia of Genes, and Genomes; PPI: Protein-Protein Interaction.

\section{Declarations}

Ethics approval and consent to participate: The study was conducted according to the guidelines of the Declaration of Helsinki, and approved by the Ethical Committee of Shanghai Sixth People's Hospital (YS2018-039 and 2018-02-24).

Consent for publication: All authors read and approved the final paper for publication.

Availability of data and materials: The datasets used and/or analyzed during the current study are available from the corresponding author on reasonable request.

Competing interests: The authors declare no conflict of interest.

Funding: This research was funded by Shanghai Municipal Health Commission, grant number 20194Y0278; Research Fund of Shanghai Sixth People's Hospital Groups, no grant number; the Shanghai Sailing Program, grant number 18YF1418700. The APC was funded by Shanghai Fengxian District Central Hospital/ Shanghai University of Medicine \& Health Sciences Affiliated Sixth People's Hospital South Campus.

Author contributions: Conceptualization, J.Z. and H.X.; methodology, D.C.; software, L.D.; validation, W.L., L.D. and J.Z.; investigation, J.Z.; resources, D.C.; data curation, J.Z.; writing-original draft preparation, J.Z.; writing-review and editing, W.L.; visualization, L.D.; supervision, H.X.; project administration, D.C.; funding acquisition, J.Z. and H.X. All authors have read and agreed to the published version of the manuscript.

Acknowledgements: We would like to thank all the researchers and study participants for their contributions.

\section{References}

[1] Ritter J, Bielack SS. Osteosarcoma. Ann Oncol 21 Suppl 7, 320-325 (2020).

[2] Balamuth NJ, Womer RB. Ewing' s sarcoma. Lancet Oncol 11, 184-192 (2010). 
[3] Burns J, Wilding CP, Jones R L \& Huang PH. Proteomic research in sarcomas - current status and future opportunities. Semin Cancer Biol 61, 56-70 (2020).

[4] Tsuda Y, Lowe M, Evans S, Parry MC, Stevenson JD, Fujiwara T, et al. Surgical outcomes and prognostic factors of non-metastatic radiation-induced sarcoma of bone. Eur J Surg Oncol 46, 293-298 (2019).

[5] Chen J, Hu Y, Zhang L, Wang Y, Wang S, Zhang Y, et al. Alginate Oligosaccharide DP5 Exhibits Antitumor Effects in Osteosarcoma Patients following Surgery. Front Pharmacol 8, 623-635 (2017).

[6] Friedman DN, Chastain K, Chou JF, Moskowitz CS, Adsuar R, Wexler LH, et al. Morbidity and mortality after treatment of Ewing sarcoma: A single-institution experience. Pediatr Blood Cancer 64, e26562-26570 (2017).

[7] Li G, Zhang P, Zhang W, Lei Z, He J, Meng J, et al. Identification of key genes and pathways in Ewing's sarcoma patients associated with metastasis and poor prognosis. Onco Targets Ther 12, 4153-4165 (2019).

[8] Cheng DD, Li J, Li SJ, Yang QC, Fan CY. CNOT1 cooperates with LMNA to aggravate osteosarcoma tumorigenesis through the Hedgehog signaling pathway. Mol Oncol 11, 388-404 (2017).

[9] Robinson MD, McCarthy DJ, Smyth GK. edgeR: a Bioconductor package for differential expression analysis of digital gene expression data. Bioinformatics 26, 139-140 (2010).

[10] Tusher VG, Tibshirani R, Chu G. Significance analysis of microarrays applied to the ionizing radiation response. Proc Natl Acad Sci USA 98, 5116-5121 (2001).

[11] Wang H, Sun Q, Zhao W, Qi L, Gu Y, Li P, et al. Individual-level analysis of differential expression of genes and pathways for personalized medicine. Bioinformatics 31, 62-68 (2015).

[12] Yan H, Cai H, Guan Q, He J, Zhang J, Guo Y, et al. Individualized analysis of differentially expressed miRNAs with application to the identification of miRNAs deregulated commonly in lung cancer tissues. Brief Bioinform 19, 793-802 (2018).

[13] Andreassen CN, Alsner J, Overgaard J. Does variability in normal tissue reactions after radiotherapy have a genetic basis-where and how to look for it? Radiother Oncol 64, 131-140 (2002).

[14] Liu C, Louhimo R, Laakso M, Lehtonen R, Hautaniemi S. Identification of sample-specific regulations using integrative network level analysis. BMC Cancer 15, 319-330 (2015).

[15] Wang W, Zhang L, Zheng K, Zhang X. miR-17-5p promotes the growth of osteosarcoma in a BRCC2dependent mechanism. Oncol Rep 35, 1473-1482 (2016). 
[16] Zhang J, Yan YG, Wang C, Zhang SJ, Yu XH, Wang WJ. MicroRNAs in osteosarcoma. Clin Chim Acta 444, 9-17 (2015).

[17] Yang J, Li YH, He MT, Qiao JF, Sang Y, Cheang LH, et al. HSP90 regulates osteosarcoma cell apoptosis by targeting the p53/TCF-1-mediated transcriptional network. J Cell Physiol 235, 3894-3904 (2019).

[18] Zhou L, Yu Y, Sun S, Zhang T, Wang M. Cry 1 Regulates the Clock Gene Network and Promotes Proliferation and Migration Via the Akt/P53/P21 Pathway in Human Osteosarcoma Cells. J Cancer 9, 2480-2491 (2018).

[19] Passacantilli I, Frisone P, De Paola E, Fidaleo M, Paronetto MP. hnRNPM guides an alternative splicing program in response to inhibition of the PI3K/AKT/mTOR pathway in Ewing sarcoma cells. Nucleic Acids Res 45, 12270-12284 (2017).

[20] Ren C, Ren T, Yang K, Wang S, Bao X, Zhang F, et al. Inhibition of SOX2 induces cell apoptosis and G1/S arrest in Ewing's sarcoma through the PI3K/Akt pathway. J Exp Clin Cancer Res 35, 44-58 (2016).

[21] Kawano M, Tanaka K, Itonaga I, Iwasaki T, Tsumura H. MicroRNA-20b promotes cell proliferation via targeting of TGF-beta receptor II and upregulates MYC expression in Ewing's sarcoma cells. Int J Oncol $51,1842-1850$ (2017).

[22] Hawkins AG, Basrur V, da Veiga Leprevost F, Pedersen E, Sperring C, Nesvizhskii Al, et al. The Ewing Sarcoma Secretome and Its Response to Activation of Wnt/beta-catenin Signaling. Mol Cell Proteomics 17, 901-912 (2018).

[23] Yang Z, Chen Y, Fu Y, Yang Y, Zhang Y, Chen Y, et al. Meta-analysis of differentially expressed genes in osteosarcoma based on gene expression data. BMC Med Genet 15, 80-87 (2014).

[24] Büttner P, Ueberham L, Shoemaker MB, Roden DM, Dinov B, Hindricks G, et al. Identification of Central Regulators of Calcium Signaling and ECM-Receptor Interaction Genetically Associated With the Progression and Recurrence of Atrial Fibrillation. Front Genet 9, 162-171 (2018).

[25] Kun-Peng Z, Chun-Lin Z, Xiao-Long M, Lei Z. Fibronectin-1 modulated by the long noncoding RNA OIP5-AS1/miR-200b-3p axis contributes to doxorubicin resistance of osteosarcoma cells. J Cell Physiol 234, 6927-6939 (2019).

[26] Zhou Y, Yin L, Li H, Liu LH, Xiao T. The LncRNA LINC00963 facilitates osteosarcoma proliferation and invasion by suppressing miR-204-3p/FN1 axis. Cancer Biol Ther 20, 1141-1148 (2019).

[27] Prideaux M, Wijenayaka AR, Kumarasinghe DD, Ormsby RT, Evdokiou A, Findlay DM, et al. SaOS2 Osteosarcoma cells as an in vitro model for studying the transition of human osteoblasts to osteocytes. Calcif Tissue Int 95, 183-193 (2014). 
[28] Jiang K, Liu H, Xie D, Xiao Q. Differentially expressed genes ASPN, COL1A1, FN1, VCAN and MUC5AC are potential prognostic biomarkers for gastric cancer. Oncol Lett 17, 3191-3202 (2019).

[29] Wang S, Zhong L, Li Y, Xiao D, Zhang R, Liao D, et al. Up-regulation of PCOLCE by TWIST1 promotes metastasis in Osteosarcoma. Theranostics 9, 4342-4353 (2019).

[30] Szklarczyk D, Morris JH, Cook H, Kuhn M, Wyder S, Simonovic M, et al. The STRING database in 2017: quality-controlled protein-protein association networks, made broadly accessible. Nucleic Acids Res 45, D362-368 (2017).

[31] Shannon P, Markiel A, Ozier O, Baliga NS, Wang JT, Ramage D, et al. Cytoscape: a software environment for integrated models of biomolecular interaction networks. Genome Res 13, 2498-2504 (2003).

\section{Figures}




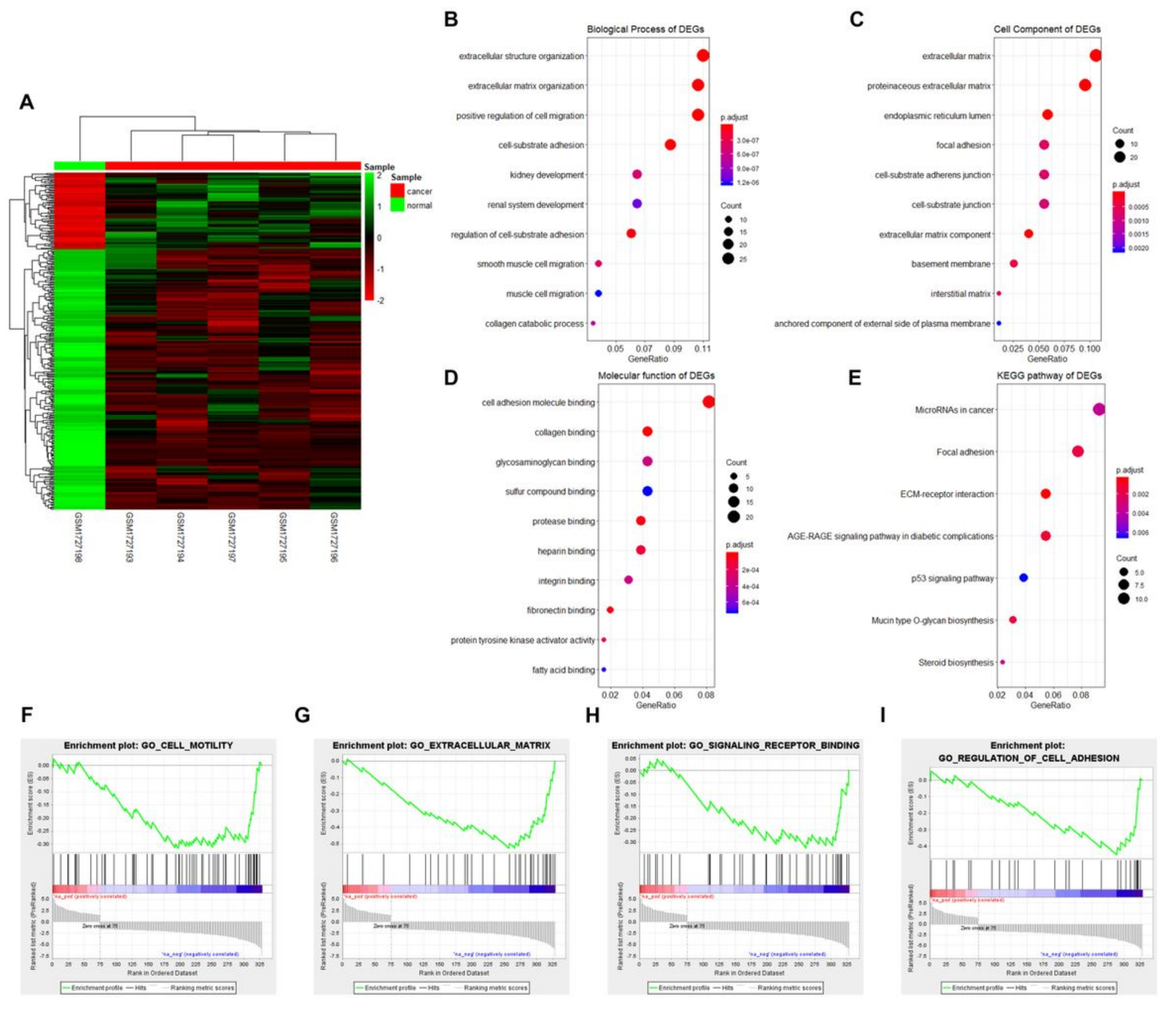

Figure 1

Identification of DEGs and their biological functions for osteosarcoma. (A) Heatmap showing 329 DEGs between osteosarcoma cells and mesenchymal stem cells. Red represents up-regulated genes and green represents down-regulated genes. GO including (B) biological process, (C) cell component and (D) molecular function and (E) KEGG enrichment results depicting underlying biological functions for these DEGs. (F-I) GSEA results based on these DEGs, including cell motility, extracellular matrix, signaling receptor binding and regulation of cell adhesion. 

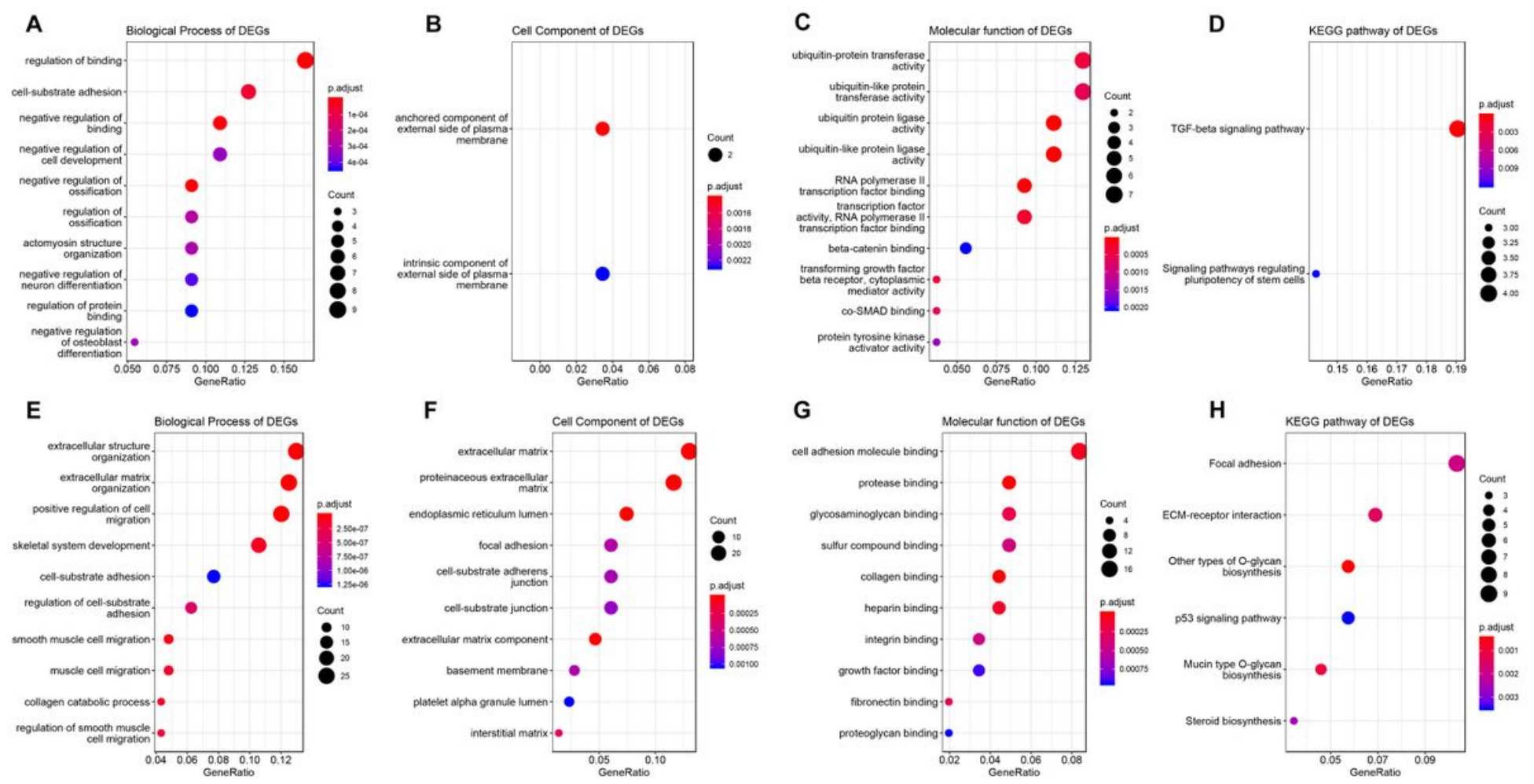

Figure 2

Functional enrichment analysis results of up- and down-regulated genes for osteosarcoma. (A) Biological processes; (B) cell component; (C) molecular function and (D) KEGG for up-regulated genes. (E) Biological processes; $(\mathrm{F})$ cell component; $(\mathrm{G})$ molecular function and $(\mathrm{H}) \mathrm{KEGG}$ for down-regulated genes. 
A
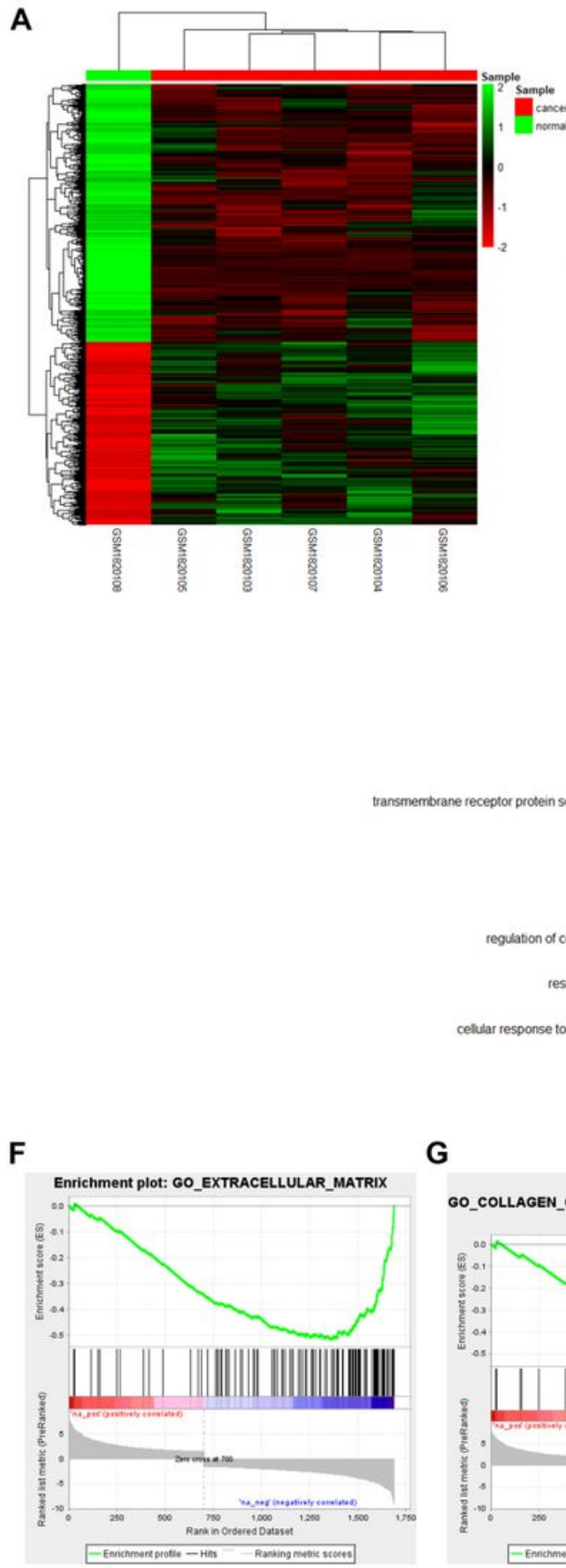

G

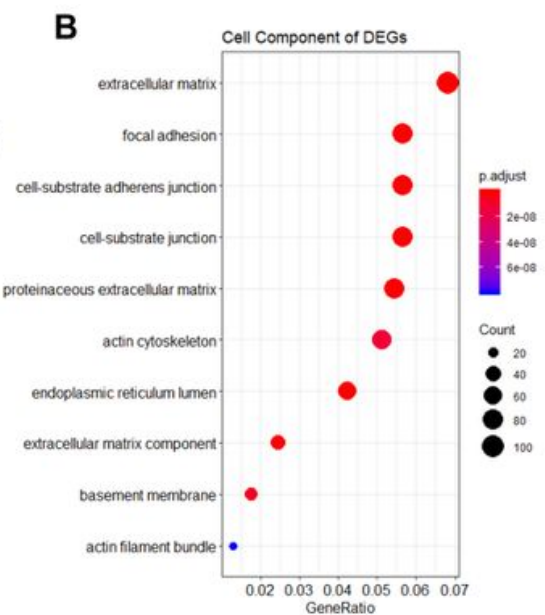

D
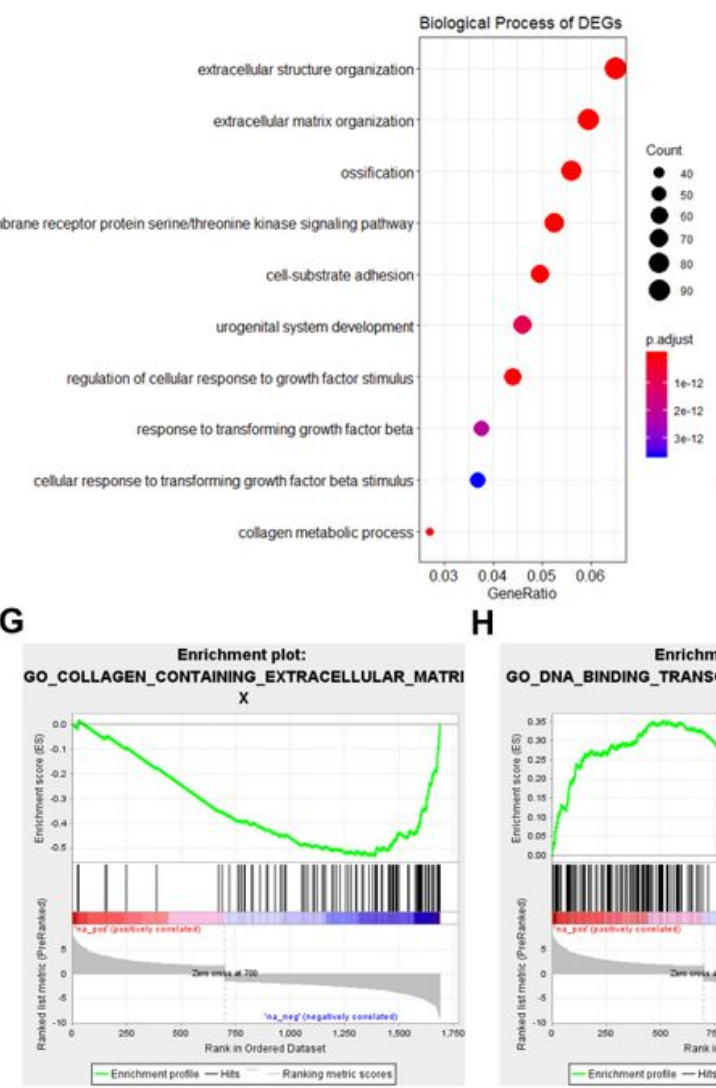

H

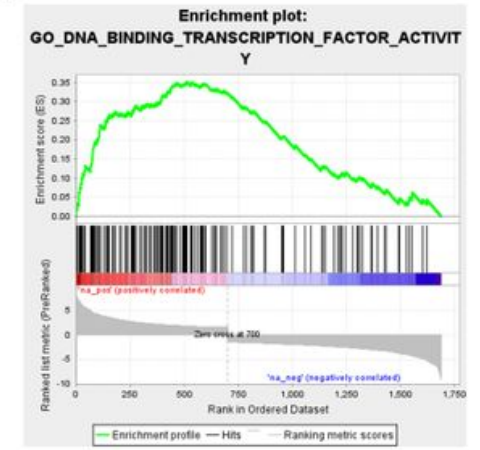

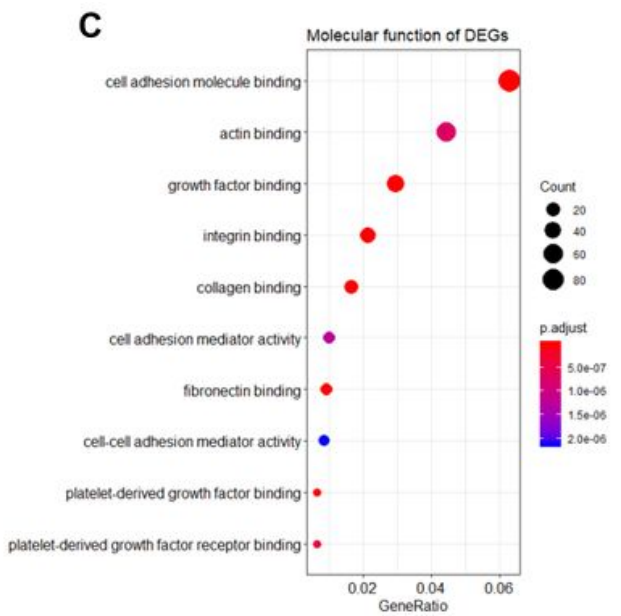

E

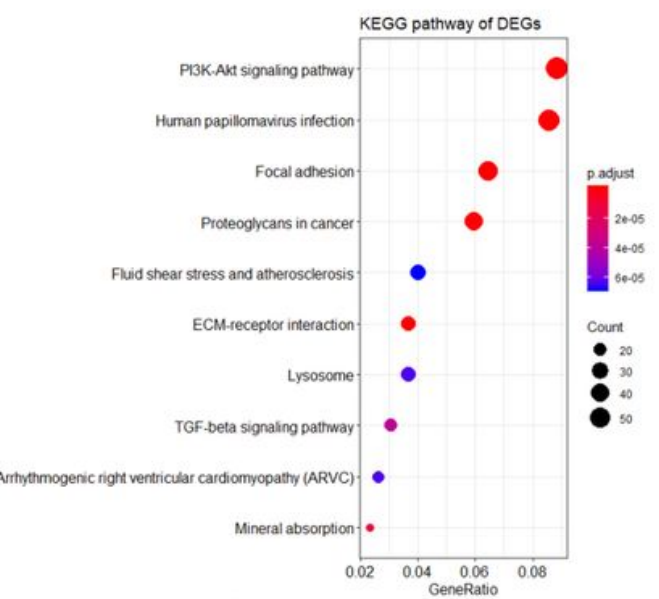

I

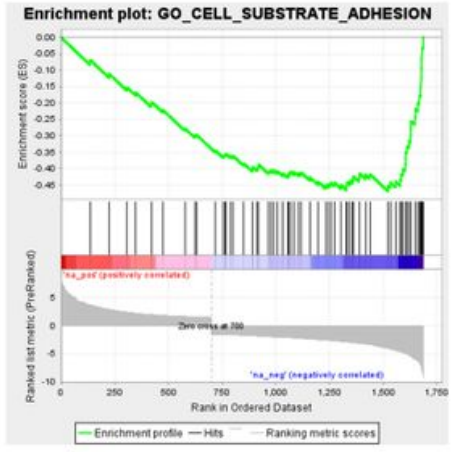

\section{Figure 3}

Identification of DEGs and their biological functions for Ewing's sarcoma. (A) Heatmap showing 1688 DEGs between Ewing's sarcoma and mesenchymal stem cells. Red represents up-regulated genes and green represents down-regulated genes. GO including (B) cell component, (C) molecular function and (D) biological process and (E) KEGG enrichment results depicting underlying biological functions for these DEGs. (F-I) GSEA results according to these DEGs, including extracellular matrix, collagen containing extracellular matrix, DNA binding transcription factor activity and cell substrate adhesion. 

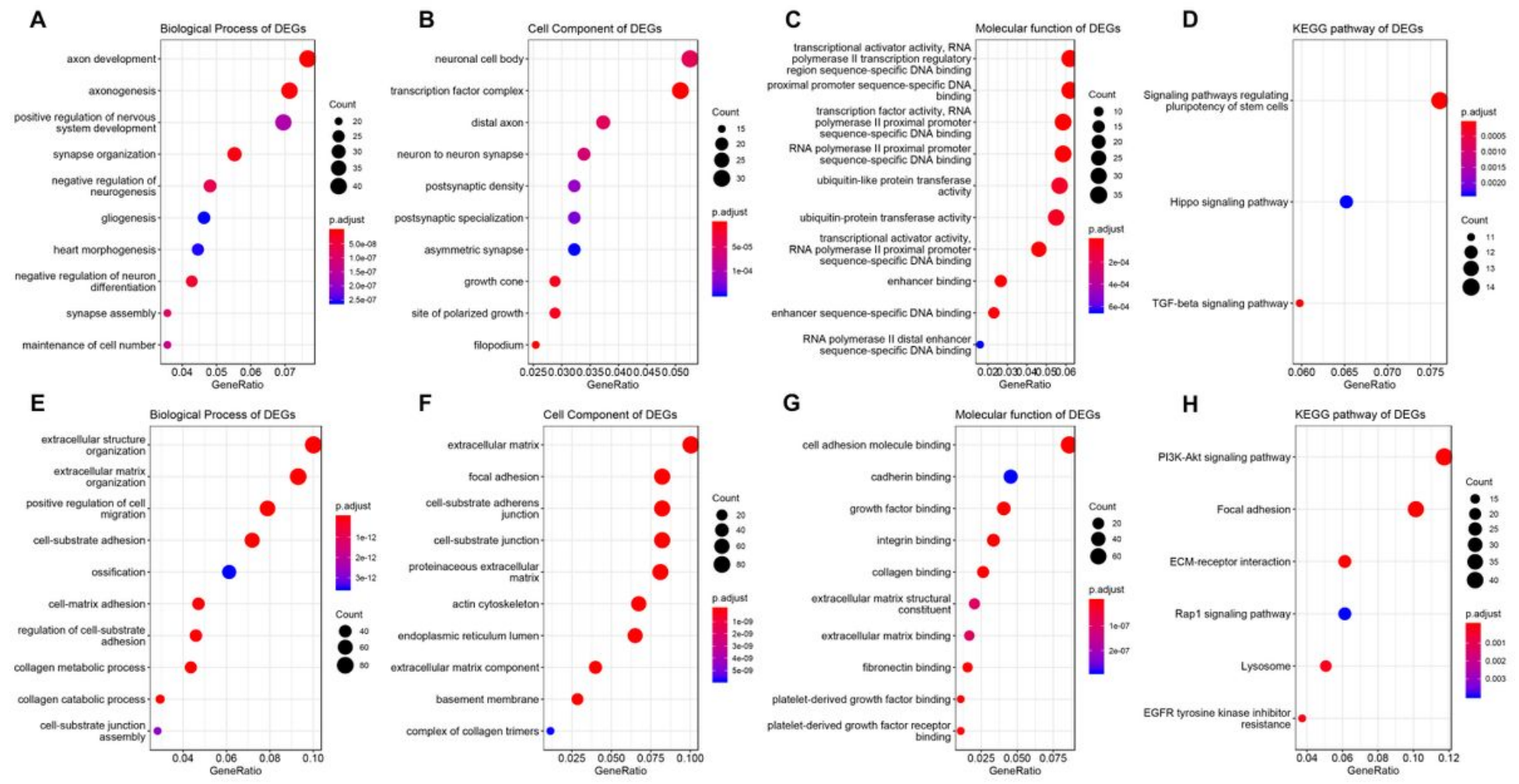

Figure 4

Functional enrichment analysis results of up- and down-regulated genes for Ewing's sarcoma. (A) Biological processes; (B) cell component; (C) molecular function and (D) KEGG for up-regulated genes. (E) Biological processes; (F) cell component; (G) molecular function and (H) KEGG for down-regulated genes. 

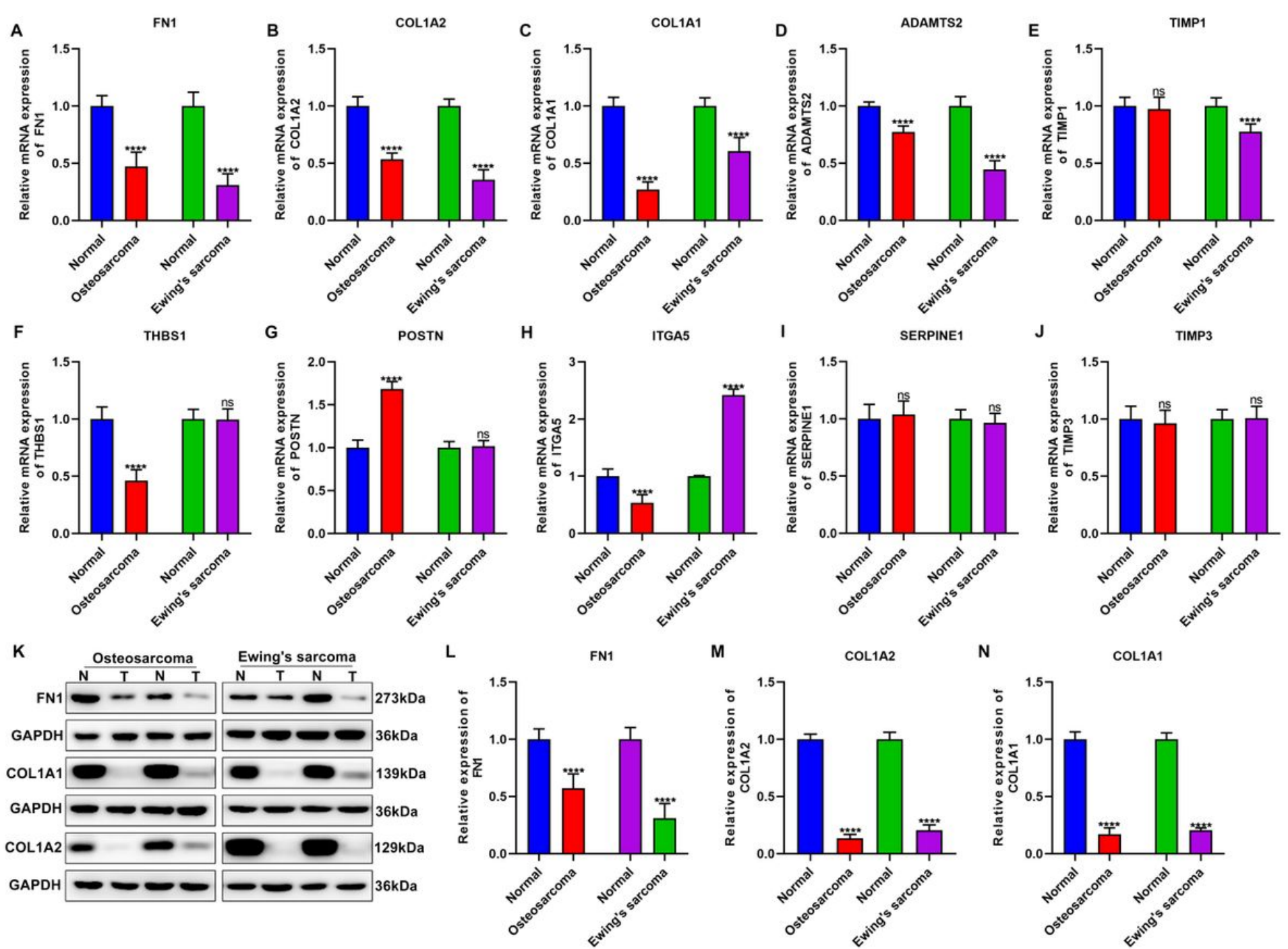

Figure 5

Validation of common oncogenic genes in osteosarcoma and Ewing's sarcoma tissues. RT-qPCR results detecting the mRNA expression of (A) FN1, (B) COL1A2, (C) COL1A1, (D) ADAMTS2, (E) TIMP1, (F) THBS1, (G) POSTN and (H) ITGA5, (I) SERPINE1 and (J) TIMP3 between osteosarcoma or Ewing's sarcoma tissues and normal tissues. (K-N) Western blot showing the expression of FN1, COL1A2 and COL1A1 proteins between osteosarcoma or Ewing's sarcoma tissues and normal tissues. Ns: not significant; $* \star \star \star p<0.0001$.

\section{Supplementary Files}

This is a list of supplementary files associated with this preprint. Click to download.

- SupplementaryFigure1.tif

- SupplementaryFigure2.tif

- SupplementaryFigure3.tif 
- SupplementaryFigure4.tif

- SupplementaryFigure5.tif

- SupplementaryFigure6.tif

- SupplementaryTable1.doc

- SupplementaryTable2.doc

- SupplementaryTable3.doc

- SupplementaryTable4.doc

- SupplementaryTable5.doc

- SupplementaryTable6.doc

- SupplementaryTable7.doc

- SupplementaryTable8.doc 